\title{
Replicability of a Millimeter-Wave Microstrip Bandpass Filter using Parallel Coupled Lines
}

Mbeutcha, Marie; Johansen, Tom Keinicke; Dong, Yunfeng; Cimoli, Bruno; Krozer, Viktor; FernandezDel-Carpio, Gonzalo M.

\section{Published in:}

Proceedings of 2018 IEEE MTT-S Latin America Microwave Conference

Link to article, DOI:

10.1109/LAMC.2018.8699012

Publication date:

2019

Document Version

Peer reviewed version

Link back to DTU Orbit

Citation $(A P A)$ :

Mbeutcha, M., Johansen, T. K., Dong, Y., Cimoli, B., Krozer, V., \& Fernandez-Del-Carpio, G. M. (Ed.) (2019). Replicability of a Millimeter-Wave Microstrip Bandpass Filter using Parallel Coupled Lines. In Proceedings of 2018 IEEE MTT-S Latin America Microwave Conference [8699012] IEEE.

https://doi.org/10.1109/LAMC.2018.8699012

\section{General rights}

Copyright and moral rights for the publications made accessible in the public portal are retained by the authors and/or other copyright owners and it is a condition of accessing publications that users recognise and abide by the legal requirements associated with these rights.

- Users may download and print one copy of any publication from the public portal for the purpose of private study or research.

- You may not further distribute the material or use it for any profit-making activity or commercial gain

- You may freely distribute the URL identifying the publication in the public portal 


\title{
Replicability of a Millimeter-Wave Microstrip Bandpass Filter using Parallel Coupled Lines
}

\author{
Marie Mbeutcha ${ }^{1}$, Tom K. Johansen ${ }^{2}$, Yunfeng Dong ${ }^{2}$, Bruno Cimoli ${ }^{2}$ and Viktor Krozer $^{2}$ \\ ${ }^{1}$ Goethe University Frankfurt, 60438 Frankfurt am Main, Germany \\ ${ }^{2}$ Technical University of Denmark, 2800 Kgs. Lyngby, Denmark \\ mbeutcha@physik.uni-frankfurt.de
}

\begin{abstract}
Replicability of filters is critical especially at millimeter-wave (mm-wave) frequencies, as a manufacturing error of a few tens of microns can significantly shift the frequency response in this range and lumped elements are not available at these frequencies. In this paper, seven replicas of a mm-wave coupled-line $3^{\text {rd }}$ order bandpass filter (BPF) are fabricated and measured under the same test conditions. The filters are designed on a single lot 10 mil-thick Rogers RO4350B substrate. The smallest spacing is $109 \mu \mathrm{m}$ and the smallest line width is $330 \mu \mathrm{m}$. Over seven replicas, the passband is from $34.8 \pm 0.2$ to $38.1 \pm 0.1 \mathrm{GHz}$; the insertion loss is $3.44 \pm 0.32 \mathrm{~dB}$; the typical return loss is $10.18 \pm 2.43 \mathrm{~dB}$. The measurement results are in accordance with the EM simulation results. They show that the reflection parameters are relatively sensitive to manufacturing tolerances and connector realization, while the transmission parameters are robust to fabrication variations. This demonstrates a satisfactory manufacturing replicability of microstrip BPF in the Ka-band (26.5-40 GHz), in the scope of radar system design.
\end{abstract}

Keywords-parallel coupled line filters, microstrip bandpass filters, millimeter-wave, replicability.

\section{INTRODUCTION}

The spectrum allocation of the $33.4-36 \mathrm{GHz}$ band by German Federal Network Agency for wind turbine structural health monitoring systems has engaged the investigation of imaging radars at mm-wave frequencies. This work proposes a simple, low-cost and replicable $3^{\text {rd }}$ order microstrip BPF that could be integrated in the transmitter of an imaging radar such as proposed in [1]. The filter is typically used to decrease the level of spurious signals such as local oscillator leakages, image frequency or unwanted harmonics.

Microstrip BPFs have the advantage of being compact, lightweight and are compatible with SMD components, but the design becomes challenging at mm-wave frequencies. Low insertion loss (IL) necessitates thin and low-loss substrates such as those based on LTCC technologies [2] or Rogers RT 5880 [3] [4]. Mm-wave coupled line filter dimensions decrease to sub-millimeter dimensions, which makes it difficult to implement via holes [2]. Furthermore, the spacing between the lines reduces greatly with respect to increasing fractional bandwidth. In [3], the authors propose a wideband BPF design solution with minimum $100 \mu \mathrm{m}$ spacing which is realizable. However, the architecture including the matching network is relatively complex, especially that mm-wave BPF design is sensitive to length variations (Fig. 1)

As an alternative, the design proposed in this paper focuses on a simple parallel coupled-line BPF layout based on Rogers RO4350B substrate. The dielectric constant is 3.48 and the loss tangent is 0.0037 , both measured at 10 $\mathrm{GHz}$. The thickness of the substrate is $0.254 \mathrm{~mm}$ with 35 $\mu \mathrm{m}$-thick copper conductor layers. To investigate the manufacturing robustness of the filter, seven replicas have
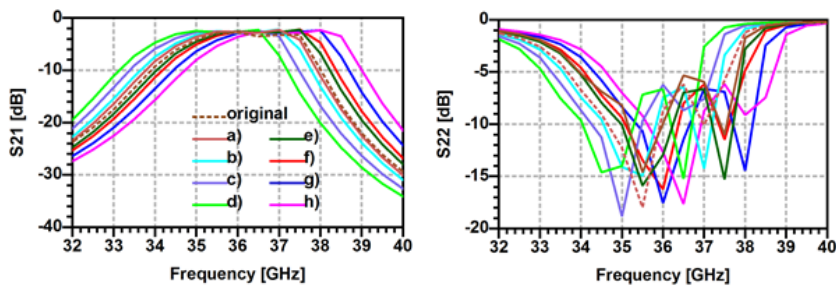

Fig. 1: Frequency response shift due to length modification for S21 and S22. The length of each coupled line has been increased by a) 5 $\mu \mathrm{m}, \mathrm{b}) 10 \mu \mathrm{m}$, c) $20 \mu \mathrm{m}$, d) $30 \mu \mathrm{m}$, and decreased by e) $5 \mu \mathrm{m}$, f) 10 $\mu \mathrm{m}, \mathrm{g}) 20 \mu \mathrm{m}, \mathrm{h}) 30 \mu \mathrm{m}$ from the original. A shift of $50 \mu \mathrm{m}$ is a typical error when manufacturing.

been fabricated externally and characterized. All replicas are built on the same substrate lot. The manufacturer guarantees a design tolerance of $80 \%$ of the filter dimension, the conductor spacing tolerance is $30 \%$. This paper aims to show that the proposed and conventional parallel coupled-line filter is realizable and replicable at mm-wave frequencies. This study is essential to ensure that the filter works as expected when integrated in a whole communication or detection system.

Section II briefly recaps the bandpass filter design procedure, while simulation results are described in Section III, and compared to the measurement results in Section IV to demonstrate the good realization and replicability of the design.

\section{FILTER DESIGN}

Let us consider a BPF of order $N$, of geometric center frequency $f_{0}=\sqrt{f_{1} f_{2}}$ and passband frequencies $f_{1}$ and $f_{2}$. The dimensions of the coupled-line BPF are determined using the classic two-step design equations derived in [5].

First the products of the $J_{i} Z_{0}$ the admittance of the $i^{t h}$ inverter $(i=1 . . N+1)$ are calculated for the $1^{\text {st }}$ coupling $J_{1} Z_{0}=\sqrt{\Delta \pi / 2 g_{1}}$, for the $n^{\text {th }}$ coupling $J_{n} Z_{0}=$ $\Delta \pi / 2 \sqrt{g_{n-1} g_{n}}$ and for the last coupling $J_{n+1} Z_{0}=$ $\sqrt{\Delta \pi / 2 g_{n} g_{n+1}}$. These products are function of the fractional bandwidth $\Delta=\left(f_{2}-f_{1}\right) / f_{0}$ and the filter prototypes $g_{i}$. Then are derived the even mode $Z_{0 e}^{i}$ and the odd mode $Z_{0 o}^{i}$ characteristic impedances for each coupled line $i: Z_{0 e}^{i}=Z_{0}\left(1+J_{i} Z_{0}+\left(J_{i} Z_{0}\right)^{2}\right)$ and $Z_{0 o}^{i}=$ $Z_{0}\left(1-J_{i} Z_{0}+\left(J_{i} Z_{0}\right)^{2}\right)$ respectively.

The physical dimensions of the coupled lines can be approximated and optimized using CAD software.

\section{FILTER SIMULATIONS}


The methodology presented in Section II is applied to design a $3^{\text {rd }}$ order Chebyshev BPF using parallel opencircuited coupled lines with $0.5 \mathrm{~dB}$ passband ripples. Given a fixed order, this filter has the advantage of having the sharpest cutoff among the classic filters [5].

The characteristic impedance is $50 \mathrm{Ohm}$. The center frequency should be $36.3 \mathrm{GHz}$; the fractional bandwidth is $10 \%$. The filter is overdesigned to $40 \mathrm{GHz}$ to compensate the frequency shift due the fringing fields at the open-circuited stubs.

The layout including the filter, $50 \mathrm{Ohm}$ transmission lines and the landing pads of $2.9 \mathrm{~mm}$ SMA connectors is predesigned and simulated using Keysight ADS. A 3D EM simulation comprising the above mentioned filter layout as well as 3D-drawing of the connectors is performed with ANSYS Electronics. In this simulation, the length of the 50 Ohm line and the tapered transition between the filter and the transmission line, as well as between the transmission line

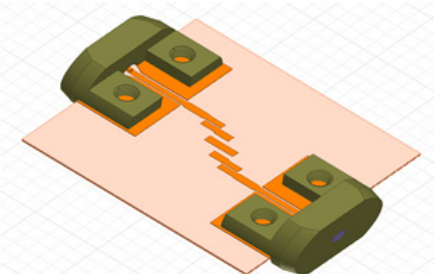

Fig. 2: Coupled-line bsand-pass filter. The outer coupled lines have a width of $417 \mu \mathrm{m}$, a spacing of $109 \mu \mathrm{m}$ and a length of $1173 \mu \mathrm{m}$. The inner coupled lines have a width of $509 \mu \mathrm{m}$, a spacing of $359 \mu \mathrm{m}$ and a length of $1136 \mu \mathrm{m}$. The filter is connected to the pads of the Hirose HK-LR-SR2 connectors

and the coplanar waveguide (CPW) structure of the connector footprint are optimized.

Figure 2 exhibits the complete design of the filter. The smallest spacing is $109 \mu \mathrm{m}$, and the smallest line width is $330 \mu \mathrm{m}$, which are reasonable for manufacturing. The SMA

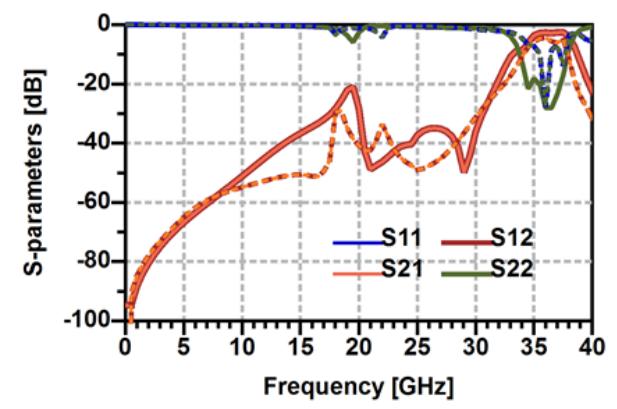

Fig. 3: Proposed filter simulations using Ansys Electronics (solid lines) and Keysight ADS (dotted lines)

connectors are Hirose HK-LR-SR2. Because they are defined from $\mathrm{DC}$ to $40 \mathrm{GHz}$, the analysis is operated within this frequency range.

Figure 3 displays the performance of the filter simulated on ADS and Ansys Electronics. For the whole filter, the $\mathrm{IL}=$ $2.5 \mathrm{~dB}$ with a $9.9 \%$ fractional bandwidth $34.5-38.1 \mathrm{GHz}$, and a return loss $>8 \mathrm{~dB}$. The simulation results are satisfactory for transmitting applications such as in [1] where the reflections are more critical than the insertion loss as the filter is integrated between two amplifiers operating in nonlinear regime.

Note that the ripples present in the stopband are due to the connector landing pads, as highlighted in Figure 4. This frequency corresponds to the main notch. The plot result
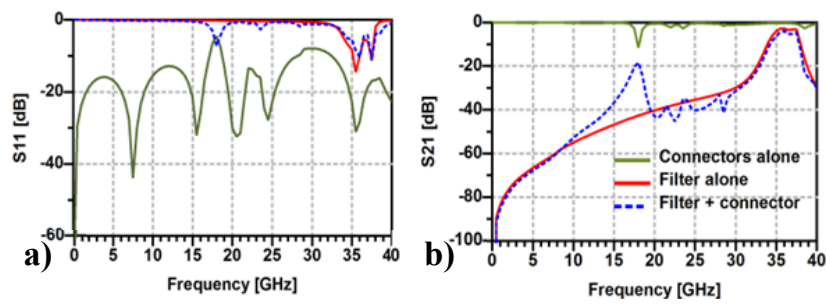

Fig. 4: ADS simulation of S11 (left) and S21 (right) comparison between a thru-line (connector landing pads and tapered $50 \mathrm{Ohm}$ transmission line), the filter alone and the full layout comprising the filter with the connectors pads

indicates that there is radiation at the transition $\mathrm{CPW}$ to microstrip.

\section{FILTER MEASUREMENTS}

Seven replicas of the microstrip filter are fabricated on the same substrate lot (see Fig. 5). For filters 1, 2, 3 and 6, the connectors landing pads are chosen to be placed at the very edge of the board. Thereby the tight design distance from the edge of PCB to the connectors via holes, recommended by the manufacturer is respected and the connectors are sure to be fixed correctly. However, dicing the PCB can generate copper burrs. In order to investigate the potential consequences of dicing process, the board is cut 200 microns away from the connector landing pads edge for filters 4, 5 and 7 (Fig. 6).
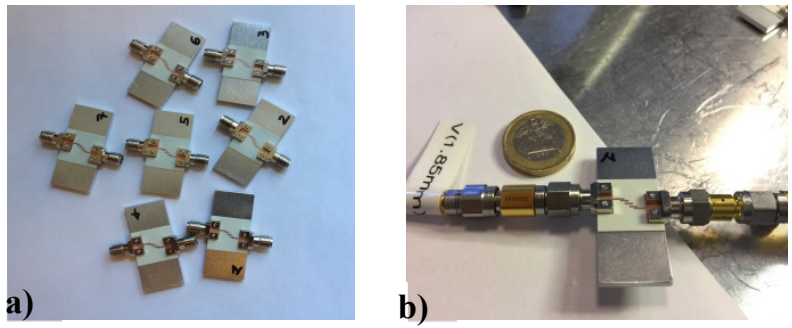

Fig. 5: a) Filter replicas b) Filter measurement setup

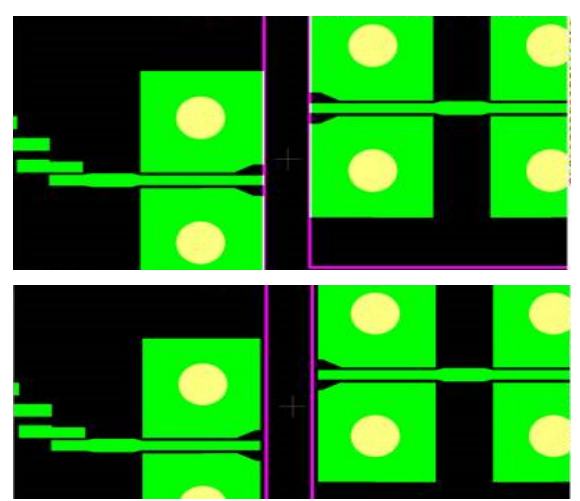

Fig. 6: In purple the PCB outline, in green the copper: a) outline at the border of the connectors landing pads, b) outline 200 microns away from the connectors landing pads

The seven filter replicas have been measured with vector network analyzer Anritsu ME7808B under the same calibration of the instrument, using calibration kit model 3652. We compare the seven replicas to each other and to the simulation results (Fig. 7). There is no substantial distortion of the frequency response as seen in Figure 1. To investigate further the feasibility and replicability of the filter, for each filter replica the center frequency, the fractional bandwidth, the insertion loss and the return loss are determined, respectively. Then, for each of these parameters the average 

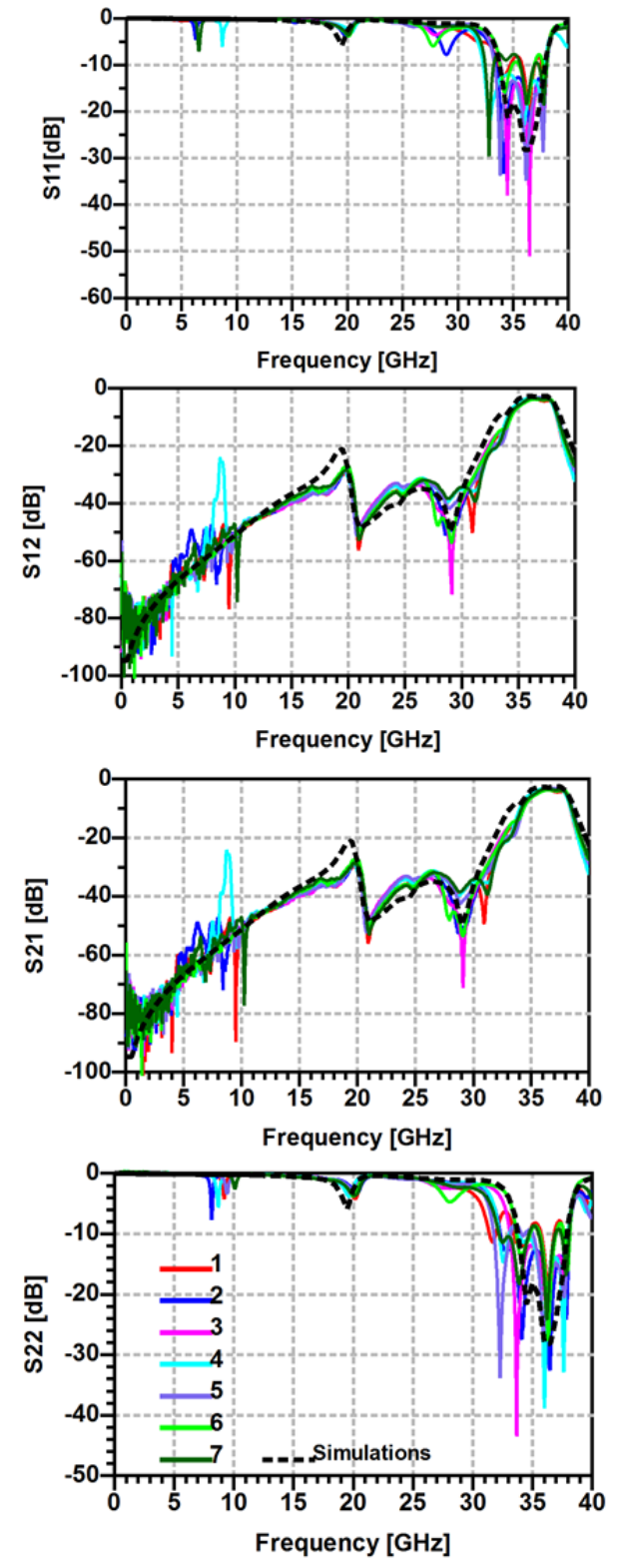

Fig. 7: Simulation and measurements results.

(AVG) and the standard deviation (SD) are calculated and summarized in Figure 8.

In Figure 8, filters 4 and 5 (with $200 \mu \mathrm{m}$ extra spacing at the PCB edge) are deviating with respect to center frequency, passband and insertion loss, unlike filters 1, 2,3 . This could be due to a defect on the connectors contact to the PCB. However, it is difficult to conclude on this phenomenon, as filters 6 and 7 do not follow the trend of their fellow filters.

In Figure 7, in the stopband, a notch appears for filter 4. This is due to a poor electrical contact of the connector, and disappeared during measurements when the connector is pressed towards the signal line. It may be due to a manufacturing error, as neither filter 5 nor 7 presents the same notches.

These general results are satisfactory and confirm the fine replicability for mm-wave coupled-line bandpass filters.
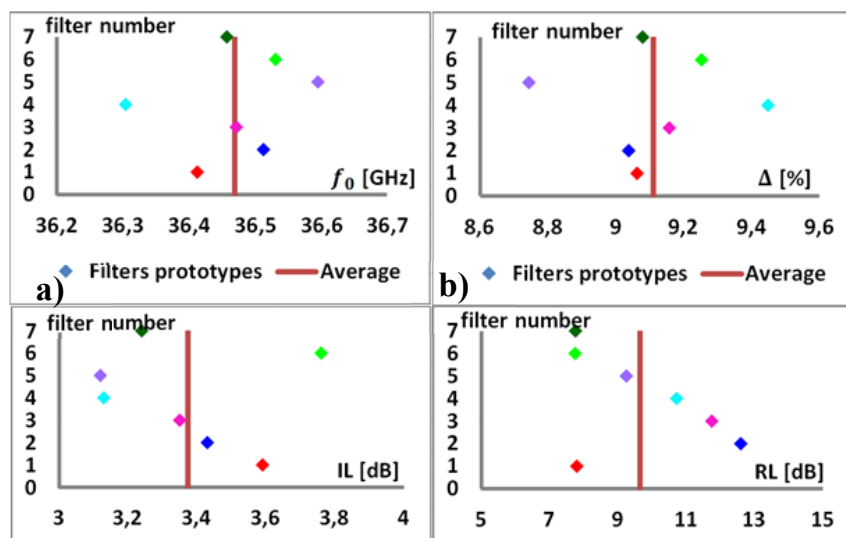

b) Filters prototypes Average

c) Filters prototypes $\longrightarrow$ Average

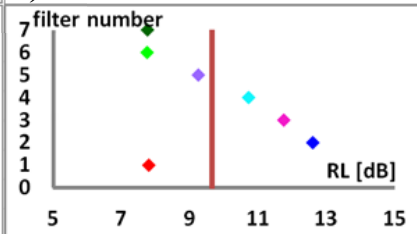

Fig. 8: Measurements results: Statistical comparison of the seven filters a) the AVG center frequency is $36.51 \mathrm{GHz}$ with $\mathrm{SD}=0.09 \mathrm{GHz}$; b) the AVG fractional bandwidth is $9.1 \%$ with $\mathrm{SD}=0.2 \%$; c) the AVG insertion loss is $3.37 \mathrm{~dB}$ with $\mathrm{SD}=0.24 \mathrm{~dB}$; d) the AVG return loss is $9.66 \mathrm{~dB}$ with $\mathrm{SD}=2.04 \mathrm{~dB}$. For transmission parameters, filters 2,3 and 7 are robust. There is no pattern with respect to reflection parameters. This demonstrates that the $\mathrm{RL}$ is sensitive to manufacturing. There is no conclusive impact regarding copper burrs.

\section{CONCLUSION}

This paper discussed the realization and replicability of a $\mathrm{mm}$-wave microstrip bandpass filter using parallel coupled lines. The filter was designed and entirely EM simulated. Seven replicas of the filter were fabricated on the same substrate lot and measured. The performance of each filter follows the same trend, and is very similar to the simulation results. It was shown that the reflection parameters are more sensitive to manufacturing tolerance than the transmission coefficients. The results of the replicability investigation of mm-wave coupled-line bandpass filter on microstrip are satisfying. This guarantees the good operation of the filter when integrated in system applications such as imaging radar system design.

\section{ACKNOWLEDGMENT}

The authors thank EU Horizon 2020 Marie SklodowskaCurie ITN CELTA project (grant agreement no. 675683) for partial financial support, RAMMS project and DLR MIMIRAWE project by the Federal Ministry for Economic Affairs and Energy (grant number: 50RA1326) for partial financial support.

\section{REFERENCES}

[1] M. Mbeutcha, G. Ulisse and V. Krozer, "Millimeter-wave imaging radar system design based on detailed system radar simulation tool," $201822^{\text {nd }}$ International Microwave and Radar Conference (MIKON), Poznan, Poland, 2018, pp. 517-520.

[2] S.W. Wong, Z. Chen and Q.-X. Chu, "Microstrip-line millimeterwave bandpass filter using interdigital coupled-line," Electronics Letters, vol. 48, no. 4, pp. 224-225, 16 February 2012.

[3] H. Zhong, R. Xu and M. Zhan, "Design for Ka-band wideband bandpass filter with three-line microstrip structures," in 2008 International Conference on Microwave and Millimeter Wave Technology, Nanjing, 2008.

[4] L. Zhang, X. Yang, Z. Yang and Z. Ma, "Design of millimeter-wave bandpass filter using microstrip stepped-impedance stubs," in IET International Communication Conference on Wireless Mobile and Computing (CCWMC 2011), Shanghai, 2011.

[5] D. M. Pozar, Microwave Engineering, $4^{\text {th }}$ Edition, New York: John Wiley \& Sons, 2011. 